\title{
ON THE DERIVATIVES OF ZETA-FUNCTIONS OF CERTAIN CUSP FORMS
}

\author{
A. LAURINČIKAS \\ Department of Mathematics and Informatics, Vilnius University, Naugarduko 24, 2006, Vilnius, Lithuania \\ Department of Physics and Mathematics, Šiauliai University, Višinskio 19, 5400, Šiauliai, Lithuania \\ e-mail: antanas.laurincikas@maf.vu.lt
}

(Received 22 January, 2004; accepted 26 July, 2004)

\begin{abstract}
The universality of the derivative and logaritmic derivative of zetafunctions of normalized eigenforms is obtained. This is applied to estimate the number of zeros of the derivative in the critical strip.
\end{abstract}

2000 Mathematics Subject Classification. 11M41.

1. Introduction. Let $F(z)$ be a normalized eigenform of weight $\kappa$. In this case $F(z)$ has the following Fourier series expansion

$$
F(z)=\sum_{m=1}^{\infty} c(m) \mathrm{e}^{2 \pi i m z}, \quad c(1)=1 .
$$

Then the function

$$
\varphi(s ; F)=\sum_{m=1}^{\infty} \frac{c(m)}{m^{s}}, \quad s=\sigma+i t,
$$

is called the zeta - function attached to the cusp form $F(z)$. The Dirichlet series for $\varphi(s ; F)$ converges absolutely in the half-plane $\sigma>\frac{\kappa+1}{2}$ and defines there a holomorphic function. It is well known that $\varphi(s ; F)$ is analytically continuable to an entire function. Moreover, for $\sigma>\frac{\kappa+1}{2}, \varphi(s ; F)$ has the Euler product expansion over primes

$$
\varphi(s ; F)=\prod_{p}\left(1-\frac{\alpha(p)}{p^{s}}\right)^{-1}\left(1-\frac{\beta(p)}{p^{s}}\right)^{-1}
$$

with $\alpha(p)+\beta(p)=c(p)$, and satisfies the functional equation

$$
(2 \pi)^{-s} \Gamma(s) \varphi(s ; F)=(-1)^{\kappa / 2}(2 \pi)^{s-\kappa} \Gamma(\kappa-s) \varphi(\kappa-s ; F) .
$$

By Deligne's estimates [2]

$$
|\alpha(p)| \leq p^{\frac{\kappa-1}{2}}, \quad|\beta(p)| \leq p^{\frac{\kappa-1}{2}} .
$$

Denote by $\mathbb{C}$ the complex plane, and let $D=\left\{s \in \mathbb{C}: \frac{\kappa}{2}<\sigma<\frac{\kappa+1}{2}\right\}$. In [8] the universality in the Voronin sense of the function $\varphi(s ; F)$ was obtained. Let $K$ be a compact subset of the strip $D$ with connected complement, and let $f(s)$ be a nonvanishing continuous on $K$ function which is analytic in the interior of $K$. Then, for 
every $\epsilon>0$,

$$
\liminf _{T \rightarrow \infty} v_{T}\left(\sup _{s \in K}|\varphi(s+i \tau ; F)-f(s)|<\epsilon\right)>0 .
$$

Here, for $T>0$,

$$
v_{T}(\ldots)=\frac{1}{T} \operatorname{meas}\{\tau \in[0 ; T]: \ldots\},
$$

where meas $\{A\}$ stands for the Lebesgue measure of the set $A \subseteq \mathbb{R}$, and in place of dots a condition satisfied by $\tau$ is to be written.

The universality is one of remarkable properties of zeta-functions. For the Riemann zeta-function this property was discovered by S. M.Voronin [9]. Later many mathematicians, among them A. Reich, S. M. Gonek, B. Bagchi, K. Matsumoto H. Mishou, R. Garunkštis, J. Steuding, W. Schwarz, R. Šleževičienė, H. Bauer, the author and others, generalized and improved the Voronin theorem for other classical zetafunctions and some classes of Dirichlet series. By the Linnik-Ibragimov conjecture, all functions in some half-plane defined by absolutely convergent Dirichlet series, analytically continuable to the left of this half-plane and satisfying some natural growth conditions are universal in the above sense.

The universality can be applied to study some analytical properties of zetafunctions, for example, the functional independence and zero-distribution. The universality property also can be used for the evaluation of complicated integrals in quantum mechanics, and in problems related to the growth order of analytic functions.

Let $K$ and $f(s)$ be as above, and let $K_{1}$ be any compact subset of $D$ included in the interior of $K$. Then a simple application of the integral Cauchy formula shows that

$$
\liminf _{T \rightarrow \infty} \nu_{T}\left(\sup _{s \in K_{1}}\left|\varphi^{\prime}(s+i \tau ; F)-f^{\prime}(s)\right|<\epsilon\right)>0 .
$$

Our aim is to obtain the universality of $\varphi^{\prime}(z ; F)$ and $\frac{\varphi^{\prime}}{\varphi}(s ; F)$ in the same form as for $\varphi(s ; F)$, and to apply this to zero-distribution of $\varphi^{\prime}(s ; F)$.

THEOREM 1. Let $K$ be a compact subset of the strip $D$ with connected complement, and let $f(s)$ be a function continuous on $K$ which is analytic in the interior of $K$. Then, for every $\epsilon>0$,

$$
\liminf _{T \rightarrow \infty} \nu_{T}\left(\sup _{s \in K}\left|\varphi^{\prime}(s+i \tau ; F)-f(s)\right|<\epsilon\right)>0 .
$$

THEOREM 2. Let $K$ and $f(s)$ be the same as in Theorem 1. Then, for every, $\epsilon>0$,

$$
\liminf _{T \rightarrow \infty} v_{T}\left(\sup _{s \in K}\left|\frac{\varphi^{\prime}}{\varphi}(s+i \tau ; F)-f(s)\right|<\epsilon\right)>0 .
$$

THEOREM 3. For every $\sigma_{1}, \sigma_{2}, \frac{\kappa}{2}<\sigma_{1}<\sigma_{2}<\frac{\kappa+1}{2}$, there exists a constant $c=$ $c\left(\sigma_{1}, \sigma_{2}\right)>0$ such that, for sufficiently large $T$, the function $\varphi^{\prime}(s ; F)$ has more than $c T$ zeros in the rectangle

$$
\sigma_{1}<\sigma<\sigma_{2}, \quad 0<t<T
$$


Theorems 2 and 3 support the Linnik-Ibragimov conjecture. On the other hand, since $\varphi^{\prime}(s ; F)$ and $\frac{\varphi^{\prime}}{\varphi}(s ; F)$ have no the Euler product over primes, the approximated function in these theorems is not necessarily non-vanishing.

Note that similar results are also valid for higher derivatives of the function $\varphi(s ; F)$.

2. A limit theorem for $\varphi^{\prime}(s ; F)$. To obtain the universality of the function $\varphi^{\prime}(s ; F)$ we need a limit theorem in the sense of the weak convergence of probability measures in the space of analytic functions. Let $G$ be a region in $\mathbb{C}$. Denote by $H(G)$ the space of analytic on $G$ functions equipped with the topology of uniform convergence on compacta. Let, for $V>0, D_{V}=\left\{s \in \mathbb{C}: \frac{\kappa}{2}<\sigma<\frac{\kappa+1}{2},|t|<V\right\}$. Denote by $\mathcal{B}(S)$ the class of Borel sets of the space $S$, and define the probability measure

$$
P_{T}(A)=v_{T}\left(\varphi^{\prime}(s+i \tau ; F) \in A\right), \quad A \in \mathcal{B}\left(H\left(D_{V}\right)\right) .
$$

We shall obtain a limit theorem with explicitly given limit measure for the measure $P_{T}$ as $T \rightarrow \infty$.

Let $\gamma=\{s \in \mathbb{C}:|s|=1\}$ be the unit circle on the complex plane, and

$$
\Omega=\prod_{p} \gamma_{p}
$$

where $\gamma_{p}=\gamma$ for each prime $p$. With product topology and pointwise multiplication the infinite - dimensional torus $\Omega$ is a compact topological Abelian group. Therefore, on $(\Omega, \mathcal{B}(\Omega))$ the probability Haar measure $m_{H}$ exists, and this leads to a probability space $\left(\Omega, \mathcal{B}(\Omega), m_{H}\right)$. Denote by $\omega(p)$ the projection of $\omega \in \Omega$ to the coordinate space $\gamma_{p}$, and on the probability space $\left(\Omega, \mathcal{B}(\Omega), m_{H}\right)$ define an $H\left(D_{V}\right)$-valued random element $\varphi^{\prime}(s, \omega ; F)$ by the formula

$$
\begin{aligned}
\varphi^{\prime}(s, \omega ; F)= & \prod_{p}\left(1-\frac{\alpha(p) \omega(p)}{p^{s}}\right)^{-1}\left(1-\frac{\beta(p) \omega(p)}{p^{s}}\right)^{-1} \\
& \times\left(-\sum_{p} \frac{\alpha(p) \omega(p) \log p}{p^{s}}\left(1-\frac{\alpha(p) \omega(p)}{p^{s}}\right)^{-1}\right. \\
& \left.-\sum_{p} \frac{\beta(p) \omega(p) \log p}{p^{s}}\left(1-\frac{\beta(p) \omega(p)}{p^{s}}\right)^{-1}\right) .
\end{aligned}
$$

Lemma 4. The probability measure $P_{T}$ weakly converges to the distribution of the random element $\varphi^{\prime}(s, \omega ; F)$ as $T \rightarrow \infty$.

Proof. We shall use a limit theorem in the space of analytic functions for the function $\varphi(s ; F)$. Such a theorem in the space $H(\widehat{D})$, where $\widehat{D}=\left\{s \in \mathbb{C}: \sigma>\frac{\kappa}{2}\right\}$, was proved in [3]. However, we consider the space $H\left(D_{V}\right)$, therefore we will apply Lemma 1 from [8]. Define on the probability space $\left(\Omega, \mathcal{B}(\Omega), m_{H}\right)$ an $H\left(D_{V}\right)$-valued random element $\varphi(s, \omega ; F)$ by the formula

$$
\varphi(s, \omega ; F)=\prod_{p}\left(1-\frac{\alpha(p) \omega(p)}{p^{s}}\right)^{-1}\left(1-\frac{\beta(p) \omega(p)}{p^{s}}\right)^{-1} .
$$


Then in $[8]$ it was obtained that the probability measure

$$
Q_{T}(A)=v_{T}(\varphi(s+i \tau ; F) \in A), \quad A \in \mathcal{B}\left(H\left(D_{V}\right)\right),
$$

converges weakly to the distribution of the random element $\varphi(s, \omega ; F)$ as $T \rightarrow \infty$.

The integral Cauchy formula shows that the function $h: H\left(D_{V}\right) \rightarrow H\left(D_{V}\right)$ defined by the formula $h(f(s))=f^{\prime}(s), f(s) \in H\left(D_{V}\right)$, is continuous.

Let $S$ and $S_{1}$ be two metric spaces, and let $g: S \rightarrow S_{1}$ be a measurable function. Then every probability measure $P$ on $(S, \mathcal{B}(S))$ induces on $\left(S_{1}, \mathcal{B}\left(S_{1}\right)\right)$ the unique probability measure $P g^{-1}$ defined by the equality $P g^{-1}(A)=P\left(g^{-1} A\right), A \in \mathcal{B}\left(S_{1}\right)$. A particular case of Theorem 5.1 of [1] asserts that if $g$ is a continuous function, $P_{n}$ and $P$ are probability measures on $(S, \mathcal{B}(S))$, and $P_{n}$ weakly converges to $P$ as $n \rightarrow \infty$, then $P_{n} g^{-1}$ also weakly converges to $P g^{-1}$ as $n \rightarrow \infty$.

Taking into account the later remark, the continuity of the function $h$ and the weak convergence of the measure $Q_{T}$, we obtain the assertion of the lemma.

3. A limit theorem for $\frac{\varphi^{\prime}}{\varphi}(s ; F)$. Let $\mathbb{C}_{\infty}$ be the Riemann sphere with spheric metric $d$ defined by the formula

$$
d\left(s_{1}, s_{2}\right)=\frac{2\left|s_{1}-s_{2}\right|}{\sqrt{1+\left|s_{1}\right|^{2}} \sqrt{1+\left|s_{2}\right|^{2}}}, \quad d(s, \infty)=\frac{2}{\sqrt{1+|s|^{2}}}, \quad d(\infty, \infty)=0,
$$

$s, s_{1}, s_{2} \in \mathbb{C}$. Denote by $M(G)$ the space of meromorphic on $G$ functions $g: G \rightarrow$ $\left(\mathbb{C}_{\infty}, d\right)$ equipped with the topology of uniform convergence on compacta. In this topology, a sequence $g_{n}(s) \in M(G)$ converges to $g(s) \in M(G)$ if

$$
d\left(g_{n}(s), g(s)\right) \rightarrow 0, \quad n \rightarrow \infty,
$$

uniformly on compact subsets of $G$.

On the probability space $\left(\Omega, \mathcal{B}(\Omega), m_{H}\right)$ define an $H\left(D_{V}\right)$-valued random element $\frac{\varphi^{\prime}}{\varphi}(s, \omega ; F)$ by

$$
\begin{aligned}
\frac{\varphi^{\prime}}{\varphi}(s, \omega ; F)= & -\sum_{p} \frac{\alpha(p) \omega(p) \log p}{p^{s}}\left(1-\frac{\alpha(p) \omega(p)}{p^{s}}\right)^{-1} \\
& -\sum_{p} \frac{\beta(p) \omega(p) \log p}{p^{s}}\left(1-\frac{\beta(p) \omega(p)}{p^{s}}\right)^{-1},
\end{aligned}
$$

and let

$$
Q_{T}(A)=v_{T}\left(\frac{\varphi^{\prime}}{\varphi}(s+i \tau ; F) \in A\right), \quad A \in \mathcal{B}\left(M\left(D_{V}\right)\right)
$$

LEMMA 5. The probability measure $Q_{T}$ weakly converges to the distribution of the random element $\frac{\varphi^{\prime}}{\varphi}(s, \omega ; F)$ as $T \rightarrow \infty$.

Proof. Let

$$
\Phi(s, \omega ; F)=\left(\varphi^{\prime}(s, \omega ; F), \varphi(s, \omega ; F)\right)
$$


Then by standard method (see, for example, [7], [6]), using Lemma 1 and Lemma 1 of [8], it can be proved that the probability measure

$$
\nu_{T}\left(\left(\varphi^{\prime}(s+i \tau), \varphi(s+i \tau)\right) \in A\right), \quad A \in \mathcal{B}\left(H^{2}\left(D_{V}\right)\right),
$$

where $H^{2}\left(D_{V}\right)=H\left(D_{V}\right) \times H\left(D_{V}\right)$, weakly converges to the distribution of the random element $\Phi(s, \omega ; F)$ as $T \rightarrow \infty$. Since

$$
d\left(\frac{1}{g_{1}}, \frac{1}{g_{2}}\right)=d\left(g_{1}, g_{2}\right),
$$

the function $h: H^{2}\left(D_{V}\right) \rightarrow M\left(D_{V}\right)$ given by the formula $h\left(g_{1}, g_{2}\right)=\frac{g_{1}}{g_{2}}, g_{1}, g_{2} \in$ $H\left(D_{V}\right)$, is continuous, the lemma follows from Theorem 5.1 of [1] and the weak convergence of the measure (2).

4. The support of the random element $\varphi^{\prime}(s, \omega ; F)$. Let $S$ be a separable metric space, and let $P$ be a probability measure on $(S, \mathcal{B}(S))$. We recall that a minimal closed set $S_{P} \subseteq S$ such that $P\left(S_{P}\right)=1$ is called a support of $P$. The set $S_{P}$ consists of all $x \in S$ such that for every neighbourhood $G$ of $x$ the inequality $P(G)>0$ is satisfied.

Let $X$ be a $S$-valued random element defined on a certain probability space $(\widehat{\Omega}, \mathcal{B}(\widehat{\Omega}), \mathbb{P})$. Then the support of the distribution $\mathbb{P}(X \in A), A \in \mathcal{B}(S)$, of $X$ is called a support of the random element $X$.

To prove the universality of $\varphi^{\prime}(s, F)$ we need the support of the random element $\varphi^{\prime}(s, \omega ; F)$.

Lemma 6. The support of the random element $\varphi^{\prime}(s, \omega ; F)$ is the whole of $H\left(D_{V}\right)$.

Proof. Let

$$
S=\left\{g \in H\left(D_{V}\right): g(s) \neq 0 \text { or } g(s) \equiv 0\right\} .
$$

Then in [8] it is proved that the support of the random element $\varphi(s, \omega ; F)$ is the set $S$. The function $h: S \rightarrow H\left(D_{V}\right)$, given by the formula $h(g(s))=g^{\prime}(s), g(s) \in S$, is continuous. Therefore, for any open set $G \subset H\left(D_{V}\right)$, we have that $h^{-1} G$ is an open set of $S$. Moreover, we note that the set $h^{-1} G$ is non empty. Note that, by Lemma 1.7.1 of [5], there exists a sequence $\left\{K_{n}\right\}$ of compact subsets of $D_{V}$ such that

$$
D_{V}=\bigcup_{n=1}^{\infty} K_{n},
$$

$K_{n} \subset K_{n+1}$ and, if $K$ is compact and $K \subset D_{V}$, then $K \subseteq K_{n}$, for some $n$. Clearly, the sets $K_{n}$ can be chosen with connected complement. For example, we can take the rectangles. Define, for $f, g \in H\left(D_{V}\right)$,

$$
\rho(f, g)=\sum_{n=1}^{\infty} 2^{-n} \frac{\rho_{n}(f, g)}{1+\rho_{n}(f, g)},
$$

where

$$
\rho_{n}(f, g)=\sup _{s \in K_{n}}|f(s)-g(s)|
$$


Then, clearly, $\rho$ is a metric in $H\left(D_{V}\right)$ that induces its topology. Hence it follows that $g$ approximates $f$ with a given accuracy in the sense of the topology of $H\left(D_{V}\right)$ if $g$ approximates $f$ with a suitable accuracy uniformly on $K_{n}$ for sufficiently large $n$. Therefore, it suffices to consider an approximation on compact subsets.

Let $f \in h^{-1} G$; then $h(f) \in G$. Let $K$ be a compact subset of $D_{V}$ with connected complement. Then by the Mergelyan theorem (see, for example, [10]) there exists a polynomial $p(s)$ which approximates $h(f(s))$ with a given accuracy uniformly on $K$. Hence we may assume that $p(s) \in G$. Therefore, we deduce that there exists a polynomial $q(s) \in h^{-1}\{p\}$ and $q(s) \neq 0$ on $D_{V}$. This shows that $h^{-1} G$ is non empty.

Now we have that

$$
m_{H}\left(\omega \in \Omega:(\varphi(s, \omega ; F))^{\prime} \in G\right)=m_{H}\left(\omega \in \Omega: \varphi(s, \omega ; F) \in h^{-1} G\right)>0,
$$

and the lemma is proved.

5. The support of the random element $\frac{\varphi^{\prime}}{\varphi}(s, \omega ; F)$. In this section we shall prove the following statement.

LEMMA 7. The support of the random element $\frac{\varphi^{\prime}}{\varphi}(s, \omega ; F)$ is the whole of $H\left(D_{V}\right)$.

Proof. We shall give a direct proof of the lemma. By the definition, $\{\omega(p)\}$ is a sequence of independent random variables. Therefore, $\left\{h_{p}(s, \omega)\right\}$, where

$$
h_{p}(s, \omega)=-\frac{\alpha(p) \omega(p) \log p}{p^{s}}\left(1-\frac{\alpha(p) \omega(p)}{p^{s}}\right)^{-1}-\frac{\beta(p) \omega(p) \log p}{p^{s}}\left(1-\frac{\beta(p) \omega(p)}{p^{s}}\right)^{-1}
$$

is a sequence of independent $H\left(D_{V}\right)$-valued random elements. The support of each $\omega(p)$ is the unit circle $\gamma$. Therefore, the support of random element $h_{p}(s, \omega)$ is the set

$$
\left\{g \in H\left(D_{V}\right): g(s)=h_{p}(s, a) \text { with } a \in \gamma\right\} .
$$

Hence by Theorem 1.7.10 of [5] the support of the random element $\frac{\varphi^{\prime}}{\varphi}(s, \omega ; F)$ is the closure of the set of all convergent series

$$
\sum_{p} h_{p}\left(s, a_{p}\right)
$$

with $a_{p} \in \gamma$. For the proof of the lemma it remains to check that the latter set is dense in $H\left(D_{V}\right)$. For this we shall apply Theorem 6.3 .10 of [5].

Let, for every fixed $p_{0}$,

$$
\widehat{h}_{p}(s, 1)= \begin{cases}h_{p}(s, 1) & \text { if } p>p_{0}, \\ 0 & \text { if } p \leq p_{0} .\end{cases}
$$

First we shall prove that the set of all convergent series

$$
\sum_{p} \widehat{a}_{p} \widehat{h}_{p}(s, 1), \quad \widehat{a}_{p} \in \gamma,
$$


is dense in $H\left(D_{V}\right)$. Clearly,

$$
h_{p}(s, 1)=-\frac{c(p) \log p}{p^{s}}+r_{p}(s),
$$

where, in view of estimates (1), the series

$$
\sum_{p} r_{p}(s)
$$

converges uniformly on compact subsets of $\widehat{D}=\left\{s \in \mathbb{C}: \sigma>\frac{\kappa}{2}\right\}$. Since

$$
\sum_{p} \frac{c^{2}(p) \log ^{2} p}{p^{2 \sigma}}<\infty
$$

for $\sigma>\frac{\kappa}{2}$, by Lemma 6.5 .3 of [5] there exists a sequence $\left\{\tilde{a}_{p}: \tilde{a}_{p} \in \gamma\right\}$ such that the series

$$
\sum_{p} \frac{c(p) \widetilde{a}_{p} \log p}{p^{s}}
$$

converges for $s \in \widehat{D}$. Therefore, by the well-known property of Dirichlet series it converges uniformly on compact subsets of $\widehat{D}$. Thus, there exists a sequence $\left\{\widetilde{a}_{p}\right.$ : $\left.\tilde{a}_{p} \in \gamma\right\}$ such that the series

$$
\sum_{p} \widetilde{a}_{p} \widehat{h}_{p}(s, 1)
$$

converges in $H\left(D_{V}\right)$. Let $g_{p}(s)=\widetilde{a}_{p} \widehat{h}_{p}(s, 1)$. Obviously, to prove the denseness of all convergent series (4) it suffices to show that the set of all convergent series

$$
\sum_{p} a_{p} g_{p}(s), \quad a_{p} \in \gamma,
$$

is dense in $H\left(D_{V}\right)$. For this we shall use Theorem 6.3.10 of [5]. We note that the series

$$
\sum_{p} g_{p}(s)
$$

converges in $H\left(D_{V}\right)$, and, for any compact $K \subset \widehat{D}$,

$$
\sum_{p} \sup _{s \in K}\left|g_{p}(s)\right|^{2}<\infty
$$

Therefore, it remains to verify the first hypothesis of Theorem 6.3.10 of [5].

Let $\mu$ be a complex-valued measure on $(\mathbb{C}, \mathcal{B}(\mathbb{C}))$ with compact support contained in $D_{V}$ such that

$$
\sum_{p}\left|\int_{\mathbb{C}} g_{p}(s) \mathrm{d} \mu(s)\right|<\infty .
$$


Define

$$
l_{p}(s)=-\frac{c(p) \widetilde{a}_{p} \log p}{p^{s}}
$$

Then we have that

$$
\sum_{p} \sup _{s \in K}\left|g_{p}(s)-l_{p}(s)\right|<\infty,
$$

for every compact $K \subset \widehat{D}$. This and (6) yield

$$
\sum_{p}\left|\int_{\mathbb{C}} l_{p}(s) \mathrm{d} \mu(s)\right|<\infty
$$

or

$$
\sum_{p}|c(p)| \log p\left|\int_{\mathbb{C}} p^{-s} \mathrm{~d} \mu(s)\right|<\infty
$$

Hence, clearly,

$$
\sum_{p}|c(p)|\left|\int_{\mathbb{C}} p^{-s} \mathrm{~d} \mu(s)\right|<\infty
$$

It was proved in $[\mathbf{8}]$ that the latter condition implies the relation

$$
\int_{\mathbb{C}} s^{m} \mathrm{~d} \mu(s)=0, \quad(m=0,1,2, \ldots) .
$$

Consequently, the first hypothesis of Theorem 6.3 .10 of [5] is also satisfied, and we have that the set of all convergent series (5) is dense in $H\left(D_{V}\right)$. As was noted above, this gives the denseness of all convergent series (4).

Now let $x_{0}(s) \in H\left(D_{V}\right), K$ be a compact subset of $D_{V}$ and $\epsilon>0$. We fix $p_{0}$ such that

$$
\sup _{s \in K}\left(1-\frac{1}{2^{\kappa / 2}}\right)^{-1} \sum_{p>p_{0}} \frac{\left(\alpha^{2}(p)+\beta^{2}(p)\right) \log p}{p^{2 \sigma}}<\frac{\epsilon}{4} .
$$

Since the set of all convergent series (4) is dense in $H\left(D_{V}\right)$, there exists a sequence $\left\{\widehat{a}_{p}: \widehat{a}_{p} \in \gamma\right\}$ such that

$$
\sup _{s \in K}\left|x_{0}(s)-\sup _{p \leq p_{0}} h_{p}(s, 1)-\sup _{p>p_{0}} \widehat{a}_{p} h_{p}(s, 1)\right|<\frac{\epsilon}{2} .
$$

Let

$$
a_{p}= \begin{cases}1 & \text { if } p \leq p_{0} \\ \widehat{a}_{p} & \text { if } p>p_{0}\end{cases}
$$


Then, taking into account (7) and (8), we find that

$$
\begin{aligned}
\sup _{s \in K}\left|x_{0}(s)-\sum_{p} h_{p}\left(s, a_{p}\right)\right| & =\sup _{s \in K}\left|x_{0}(s)-\sum_{p \leq p_{0}} h_{p}(s, 1)-\sup _{p>p_{0}} h_{p}\left(s, \widehat{a}_{p}\right)\right| \\
& \leq \sup _{s \in K}\left|x_{0}(s)-\sum_{p \leq p_{0}} h_{p}(s, 1)-\sup _{p>p_{0}} \widehat{a}_{p} h_{p}(s, 1)\right| \\
& +\sup _{s \in K}\left|\sum_{p>p_{0}} \widehat{a}_{p} h_{p}(s, 1)-\sup _{p>p_{0}} h_{p}\left(s, \widehat{a}_{p}\right)\right| \\
& <\frac{\epsilon}{2}+\sup _{s \in K} 2\left(1-\frac{1}{2^{\kappa / 2}}\right)^{-1} \sum_{p>p_{0}} \frac{\left(\alpha^{2}(p)+\beta^{2}(p)\right) \log p}{p^{2 \sigma}}<\epsilon .
\end{aligned}
$$

This shows the denseness of the set of all convergent series (3). Therefore, the closure of this set is the whole of $H\left(D_{V}\right)$, and the lemma is proved.

6. Proof of Theorems 1, 2 and 3. Proof of Theorem 1. Obviously, there exists $V>0$ such that $K \subset D_{V}$. First we suppose that $f(s)$ has analytic continuation to $D_{V}$. Denote by $G$ the set of functions $g \in H\left(D_{V}\right)$ such that

$$
\sup _{s \in K}|g(s)-f(s)|<\epsilon .
$$

By Lemma 3 the function $f(s)$ is contained in the support of the random element $\varphi^{\prime}(s, \omega ; F)$. Since the set $G$ is open, Lemma 1 and the properties of weak convergence [1] and support yield

$$
\liminf _{T \rightarrow \infty} \nu_{T}\left(\sup _{s \in K}|\varphi(s+i \tau ; F)-f(s)|<\epsilon\right) \geq m_{H}\left(\omega \in \Omega: \varphi^{\prime}(s, \omega ; F) \in G\right)>0 .
$$

Now let $f(s)$ be as in the statement of Theorem 1. By the Mergelyan theorem (see, for example, [10]) there exists a polynomial $p_{n}(s)$ such that

$$
\sup _{s \in K}\left|f(s)-p_{n}(s)\right|<\frac{\epsilon}{2} .
$$

From the first part of the proof we have that

$$
\liminf _{T \rightarrow \infty} v_{T}\left(\sup _{s \in K}\left|\varphi(s+i \tau ; F)-p_{n}(s)\right|<\frac{\epsilon}{2}\right)>0 .
$$

This and (9) prove the theorem.

Proof of Theorem 2. The proof is similar to that of Theorem 1 and uses Lemmas 2 and 4.

Proof of Theorem 3. Let

$$
\widehat{\sigma}=\frac{\sigma_{1}+\sigma_{2}}{2}, \quad \sigma_{0}=\max \left(\left|\sigma_{1}-\frac{2 \kappa+1}{4}\right|,\left|\sigma_{2}-\frac{2 \kappa+1}{4}\right|\right)
$$


and $f(s)=s-\widehat{\sigma}$. Suppose that $0<\epsilon<\frac{\sigma_{2}-\sigma_{1}}{20}$. Then, by Theorem 1 , there exists a constant $c=c\left(\sigma_{1}, \sigma_{2}\right)>0$ such that for sufficiently large $T$

$$
v_{T}\left(\max _{\left|s-\frac{2 \kappa+1}{4}\right| \leq \sigma_{0}}\left|\varphi^{\prime}(s+i \tau ; F)-f(s)\right|<\epsilon\right)>c .
$$

The circle $|s-\widehat{\sigma}|=\frac{\sigma_{2}-\sigma_{1}}{2}$ is contained in the disc $\left|s-\frac{2 \kappa+1}{4}\right| \leq \widehat{\sigma}$, therefore for $\sigma$ satisfying (10)

$$
\max _{|s-\widehat{\sigma}|=\frac{\sigma_{2}-\sigma_{1}}{2}}\left|\varphi^{\prime}(s+i \tau ; F)-(s-\widehat{\sigma})\right|<\frac{\sigma_{2}-\sigma_{1}}{20} .
$$

This shows that the functions $(s-\widehat{\sigma})$ and $\varphi^{\prime}(s+i \tau ; F)-(s-\widehat{\sigma})$ in the disc $|s-\widehat{\sigma}|=$ $\frac{\sigma_{2}-\sigma_{1}}{2}$ satisfy the hypotheses of the Rouche theorem. However, the function $(s-\widehat{\sigma})$ in the interior of the disc $|s-\widehat{\sigma}|=\frac{\sigma_{2}-\sigma_{1}}{2}$ has precisely one zero, therefore by Rouché's theorem the function $\varphi^{\prime}(s+i \tau ; F)$ also has in this disc one zero. Since the number of such $\tau \in[0, T]$ by (10) is greater than $c T$, the theorem is proved.

The author thanks the referee for very useful suggestions.

ACKNOWLedgements. The work was partially supported by a grant from the Lithuanian Foundation of Studies and Science.

\section{REFERENCES}

1. P. Bilingsley, Convergence of probability measures (John Wiley and Sons, New York, 1968).

2. P. Deligne, La conjecture de Weil I, Publ. I.H.E.S., 43 (1974), 273-307.

3. A. Kačenas and A. Laurinčikas, On Dirichlet series related to certain cusp forms, Liet. Matem. Rink. 38 (1998), 82-97 (in Russian) and Lith. Math. J. 38 (1998), 64-76.

4. A. A. Karatsuba and S. M. Voronin, The Riemann zeta-function (de Gruyter, 1990).

5. A. Laurinčikas, Limit theorems for the Riemann zeta-function (Kluwer Academic Publishers, 1996).

6. A. Laurinčikas and R. Garunkštis, The Lerch zeta-function (Kluwer Academic Publishers, 2002).

7. A. Laurinčikas and K. Matsumoto, Joint value-distribution theorems on Lerch zetafunctions, Liet. Matem. Rink 38 (1998), 312-326 and Lith. Math. J. 38 (1998), 238-249.

8. A. Laurinčikas and K. Matsumoto, The universality of zeta-functions attached to certain cusp forms, Acta Arith. 98 (2001), 345-359.

9. S. M. Voronin, Theorem on the "universality" of the Riemann zeta-function, Izv. Akad. Nauk SSSR, Ser. matem. 39 (1975), 475-486 (in Russian) and Math. USSR Izv 9 (1975), 443-453.

10. J. L. Walsh, Interpolation and approximation by rational functions in the complex domain (Amer. Math. Soc. Coll. Publ., 1960). 